\title{
Molecular Heterogeneity of Papillary Thyroid Cancer: Comparison of Primary Tumors and Synchronous Metastases in Regional Lymph Nodes by Mass Spectrometry Imaging
}

\author{
Marta Gawin $^{1}$ (D) - Agata Kurczyk ${ }^{1}$ (D) - Ewa Stobiecka ${ }^{1}$ (D) Katarzyna Frątczak $^{2}$ (D) Joanna Polańska ${ }^{2}$ (D) \\ Monika Pietrowska ${ }^{1}$ (D) Piotr Widłak $^{1}$ (D)
}

Published online: 300 ctober 2019

(C) The Author(s) 2019

\begin{abstract}
Intra-tumor heterogeneity results from both genetic heterogeneity of cancer (sub)clones and phenotypic plasticity of cancer cells that could be induced by different local microenvironments. Here, we used mass spectrometry imaging (MSI) to compare molecular profiles of primary tumors located in the thyroid gland and their synchronous metastases in regional lymph nodes to analyze phenotypic heterogeneity in papillary thyroid cancer. Two types of cancerous (primary tumor and metastasis) and two types of not cancerous (thyroid gland and lymph node) regions of interest (ROIs) were delineated in postoperative material from 11 patients, then the distribution of tryptic peptides (spectral components) was analyzed by MSI in all tissue regions. Moreover, tryptic peptides identified by shotgun proteomics in corresponding tissue lysates were matched to components detected by MSI to enable their hypothetical protein annotation. Unsupervised segmentation of all cancer ROIs revealed that different clusters dominated in tumor ROIs and metastasis ROIs. The intra-patient similarity between thyroid and tumor ROIs was higher than the intra-patient similarity between tumor and metastasis ROIs. Moreover, the similarity between tumor and its metastasis from the same patients was lower than similarities among tumors and among metastases from different patients (inter-patient similarity was higher for metastasis ROIs than for tumor ROIs). Components differentiating between tumor and its metastases were annotated as proteins involved in the organization of the cytoskeleton and chromatin, as well as proteins involved in immunity-related functions. We concluded that phenotypical heterogeneity between primary tumor and lymph node metastases from the same patient was higher than inter-tumor heterogeneity between primary tumors from different patients.
\end{abstract}

Keywords Intratumor heterogeneity $\cdot$ Lymph node $\cdot$ Mass spectrometry imaging $\cdot$ Metastasis $\cdot$ Proteomics $\cdot$ Thyroid cancer

\section{Introduction}

Tumor heterogeneity is a crucial phenomenon involved in the natural history of cancer affecting the response to treatment. Genetic heterogeneity in individual cancer is a result of

Marta Gawin and Agata Kurczyk contributed equally to this work.

Electronic supplementary material The online version of this article (https://doi.org/10.1007/s12022-019-09593-2) contains supplementary material, which is available to authorized users.

Piotr Widłak

piotr.widlak@io.gliwice.pl

1 Maria Skłodowska-Curie Institute-Oncology Center, 44-101 Gliwice, Poland

2 Data Mining Division, Faculty of Automatic Control, Electronics and Computer Science, Silesian University of Technology, 44-100 Gliwice, Poland evolution characterized by (usually) a monoclonal origin and poly(sub)clonal progression, which involves the accumulation of genetic alterations. As a result, solid cancers evolve into mosaic entities composed of a mixture of cells with different genomes. Intra-tumor heterogeneity could be hypothetically observed in all phenotypic features, including cellular morphology, gene and protein expression, metabolism, and metastatic potential. Tumor heterogeneity observed at the level of a phenotype could be "hereditary" in nature and result from genetic (and epigenetic) heterogeneity. However, a substantial component of phenotypic tumor heterogeneity could be related to "non-hereditary" factors. These non-hereditary components involve differentiation of cancer stem cells, epidermal to mesenchymal transition, and phenotypic plasticity induced by interactions between cancer cells and different local microenvironments. Moreover, tumor heterogeneity is further increased by the presence of heterotypic elements, including immune cells, connective tissues, microvasculature, etc. 
Furthermore, the divergence between a primary tumor and a metastatic outgrowth is another important aspect of tumor heterogeneity [1-3].

Cancer is a systemic disease, where a large number of cells is shed into the bloodstream and lymph vessels at some stage of development, some of which settle down in distinct sites and develop into metastases. Distant metastasis is responsible for the majority of cancer-related deaths; hence, understanding the heterogeneity of metastatic cancers is an important issue to address. Therefore, molecular testing based on metastases-derived specimens is an emerging aspect of cancer diagnostics. Different models of a metastatic spread could be proposed, assumed that the acquisition of a metastatic potential is the final step of cancer progression or that acquiring the ability of a metastatic spread could be an early event during cancer development characteristic for a small subclone of the primary tumor, which could imply either high or low genetic similarity between the primary site and metastasis, respectively [4]. Though general conclusiveness of experimental evidence is rather limited, the available data on genetic and molecular similarities between a primary tumor and distant metastases could support either possibility [5-8]. It is still a matter of debate whether cancer cells first form metastases in (regional) lymph nodes then subsequently disseminate further (possibly after acquiring additional features), or simultaneously spread from a primary tumor to regional lymph nodes and distant sites. Nevertheless, the molecular characteristics of cancer cells settled in regional lymph nodes remains a valuable potential diagnostic and prognostic feature [9]. Different degrees of genetic heterogeneity (mutations and chromosomal aberrations) were reported between primary tumor and different lymph node metastases in colorectal cancer [10, 11], melanoma [12] and thyroid cancer $[13,14]$. Moreover, a few works reported differences between a primary tumor and lymph node metastases with respect to the expression of alternative transcripts [15] and the expression of receptors like HER2 [16]. Nonetheless, data on molecular heterogeneity between a primary tumor and cancer cells present in lymph nodes are rather incomplete, which restricts their general impact.

Despite the fundamental importance of intra-tumor heterogeneity, surprisingly few experimental data were collected with direct relevance to this phenomenon over decades, which is a consequence of the serious limitations of analytical approaches that could be implemented in this field. In general, two major approaches were used in the studies of molecular heterogeneity of solid cancers. The first approach is based on imaging methods that could analyze selected factors in a "continuous" mode, which included analysis of target genes (by fluorescent in situ hybridization) or proteins (by immunohistochemistry) in a specific morphological context. The second approach is based on analysis of material derived from a few physically separated sub-regions of a tumor (e.g., multiple biopsies) [17] or even single cells isolated from a tumor [18]. This strategy allows for global characterization of the genetic and molecular profile of tumor sub-regions using multiple "omics" approaches, yet the possibility to place the resulting data in a specific histological context is rather limited (or even lost in the case of current single-cell sequencing approaches). Therefore, mass spectrometry imaging (MSI), which enables an analysis of a complete molecular profile in a spatially continuous manner and a close correlation of a molecular map with histopathological features of a tissue, appears the best available method to study the phenotypic heterogeneity of cancer [19-22]. Here, we used the matrixassisted laser desorption/ionization mass spectrometry imaging (MALDI-MSI) approach to analyze molecular phenotypic heterogeneity in papillary thyroid cancer (PTC) and compared molecular profiles of primary tumors located in the thyroid gland and synchronous metastases of cancer in regional lymph nodes. Unexpectedly, we provided direct evidence that phenotypical intra-tumor heterogeneity between primary tumor and lymph node metastases from the same patient was higher than inter-tumor heterogeneity between primary tumors from different patients.

\section{Materials and Methods}

\section{Clinical Material}

Postoperative tissue was collected during thyroidectomy and simultaneous lymphadenectomy (surgery was the first therapeutic intervention in all cases), then stored as formalin-fixed paraffin-embedded (FFPE) material. Samples derived from 11 patients (10 females; aged 17-71, median age 44) with papillary thyroid carcinoma, PTC (stages pT: 1a-2, pN 1a-1b) treated at Maria Skłodowska-Curie Institute-Oncology Center in Gliwice between 2014 and 2016 were used in the study. The study was approved by the appropriate local Ethics Committee (approval no. KB/430-17/13). Tissue material was re-inspected by an experienced pathologist before the study; cancer regions of interests (ROI) were delineated in both thyroid glands and lymph nodes.

\section{Sample Preparation for MALDI-MSI}

FFPE tissue blocks were sectioned into $6 \mu \mathrm{m}$ sections using an HM 340E rotary microtome (Thermo Fisher Scientific, Waltham, MA, USA). For each patient (p1-through p11), a set of FFPE tissue sections (one from the primary location and at least three from lymph nodes) was placed on a separate ITO glass slide (Bruker Daltonik, Bremen, Germany) covered with poly-L-lysine; a mixture of poly-L-lysine solution $0.1 \%$ $(\mathrm{w} / \mathrm{v})$ in $\mathrm{H}_{2} \mathrm{O}$ with $0.2 \%(\mathrm{v} / \mathrm{v})$ water solution of IGEPAL® CA-630 (both from Sigma-Aldrich) in a volume ratio of 1:1 was used for ITO glass slide coating. Slides were subsequently subjected to thermal treatment $\left(37^{\circ} \mathrm{C}\right.$ for $18 \mathrm{~h}$ followed by 
$1 \mathrm{~h}$ at $60^{\circ} \mathrm{C}$ ) in order to increase adherence of tissue sections to the slide surface. Paraffin was removed from sections by consecutive washing in xylene 5 min (twice), ethanol $99.8 \% 5$ min, ethanol $96 \% 5 \mathrm{~min}$, ethanol $50 \% 5 \mathrm{~min}$, then glass slides were dried on air. Heat-induced antigen retrieval was performed in $10 \mathrm{mM}$ Tris- $\mathrm{HCl} \mathrm{pH} 9.0$ for $20 \mathrm{~min}$ at $95^{\circ} \mathrm{C}$ using a StableTemp ${ }^{\mathrm{TM}}$ water bath (Cole Palmer Instruments Co., Chicago, IL, USA). The solution was then cooled down for $20 \mathrm{~min}$ at room temperature, slides were washed in water for 1 min, dried and placed in a vacuum desiccator for $15 \mathrm{~min}$. Trypsin solution $\left(20 \mu \mathrm{g} / \mathrm{mL}\right.$ in $\left.25 \mathrm{mM} \mathrm{NH}_{4} \mathrm{HCO}_{3}\right)$ was uniformly sprayed over the whole glass slide with the use of SunCollect micro fraction collector/MALDI spotter (SunChrom GmbH, Friedrichsdorf, Germany) operated in the pneumatic sprayer mode, according to the method of Heijs et al. [23], resulting in deposition of $0.006 \mu \mathrm{g}$ of trypsin per square millimeter. This was followed by incubation at 37 ${ }^{\circ} \mathrm{C}$ for $18 \mathrm{~h}$ in a humid chamber. Sequencing Grade Modified Trypsin from Promega (Madison, WI, USA) was used in the study. An optical image (2400 dpi) was registered for a glass slide with marked fiducials, and a tissue section was coated with matrix solution $(5 \mathrm{mg} / \mathrm{mL} \alpha$-cyano-4-hydroxycinnamic acid in $50 \%$ ACN, $0.3 \%$ TFA) with the use of SunCollect device according to [23], resulting in deposition of $3.8 \mu \mathrm{g}$ of matrix per square millimeter.

\section{MALDI-MSI Measurements}

Prior to automatic measurements, the spectrometer was externally calibrated with the use of Peptide Calibration Standard II (Bruker Daltonik, Bremen, Germany). Spectra of tryptic peptides were acquired using an ultrafleXtreme MALDI-TOF/ TOF mass spectrometer (Bruker Daltonik, Germany) equipped with a smart-beam II $^{\mathrm{TM}}$ laser operating at $1 \mathrm{kHz}$ repetition rate working in positive reflectron mode within $\mathrm{m} /$ $\mathrm{z}$ range of 700-3700, with laser focus diameter of 4 large and $100 \mu \mathrm{m}$ raster width. Ions were accelerated at $25 \mathrm{kV}$ with a PIE time delay of 100 ns. Four hundred shots were collected from each laser position with a random walk on (40 shots at a raster spot). After imaging, the matrix was washed off the glass slides with $70 \%$ ethanol (two washes, 1 min each), and the sections were stained with hematoxylin and eosin, then scanned and used for image co-registration (using flexImaging software). Compass for flex 1.4 software package (Bruker Daltonik, Bremen, Germany) was used for spectra acquisition and handling.

\section{Spectra Processing and Identification of Spectral Components}

Standard spectrum preprocessing sequenced steps were applied as follows: (i) resampling to common mass channels, (ii) adaptive baseline detection and correction [24], (iii) outlying spectra identification according to TIC value using Bruffaerts' criterion [25], (iv) fast Fourier transform-based spectral alignment [26], and (v) TIC normalization. The Gaussian mixture model (GMM) approach described in detail elsewhere [27, 28] was used for the average spectrum modeling and peak detection. Peptide abundance was estimated by pairwise convolution of the GMM components and individual spectra. Spectrum post-processing procedure was applied to reduce the data dimensionality by filtering out GMM components of high variance and low amplitude. GMM components modeling the same spectrum peak were identified and merged. The resulting dataset featured 2696 components detected in $\mathrm{m} / \mathrm{z}$ range between 699 and 3430 that were termed molecular components hereafter, which represent tryptic peptide species imaged by MSI.

\section{MSI Data Analysis}

To assess the similarity between different ROIs of tissue samples, the pairwise similarity index [29] was calculated. Spectra were labeled according to their location in tissue specimens of 11 patients (p1-p11) and 1 of 4 possible tissue histopathological types (thyroid tumor, metastasis, normal thyroid, normal lymph node) creating 44 spectra subsets. Similarity index was calculated between or within spectra subsets in three different manners: (i) within the same type of ROI and within the same patient (intra-patient), (ii) between different types of ROI within the same patient (intra-patient), and (iii) within the same type of ROI among different patients (inter-patient). Cohen's effect size based on mean and pooled standard deviation [30] was calculated as a quantitative measure of the magnitude of differences in the abundance of each molecular component between different ROIs. Unsupervised molecular image segmentation was performed for cancer tissue regions (primary thyroid tumor and metastasis) for all 11 tissue specimens together by the deglomerative divisive iK-means algorithm [31, 32]. The algorithm's stop criterion was adjusted to create clusters of size not less than 40 spectra included (the assumption results from a relatively small number of spectra creating metastases ROI in a single tissue specimen).

\section{LC-MALDI MS/MS Analysis and Identification of Molecular Components}

Representative samples of the cancerous thyroid gland (ca. $60 \%$ of cancer cells) and lymph node with cancer spread (ca. $20 \%$ of cancer cells) were used for protein identification using the shotgun LC-MS/MS approach. Protein lysates were prepared and subjected to tryptic digestion according to a modified version of a combination of FASP with Stage-Tip fractionation as described in detail elsewhere [33]. Tryptic peptides were then separated using an EASY-nLC nano-liquid chromatograph (Proxeon) coupled with PROTEINEER fc II 
fraction collector (Bruker) and analyzed using ultrafleXtreme MALDI-TOF/TOF mass spectrometer. A detailed description of instrumental settings of the LC-MALDI-MS/MS system is given in [33]. Registered MS/MS spectra were exported to ProteinScape 3.1 software (Bruker Daltonik) and analyzed using Mascot Server 2.5.1 (Matrix Science, London, UK); for details, see [33]. The hypothetical identity of molecular components detected in MSI was established via assignment of their $\mathrm{m} / \mathrm{z}$ values to measured masses of peptides identified in LC-MALDI-MS/MS experiment; the assignment was performed allowing $\pm 0.05 \%$ mass tolerance (components overrepresented in lymph nodes were annotated in the list of peptides identified in specimens of metastasis-containing lymph nodes while components overrepresented in thyroid and cancer were annotated in the list of peptides identified in specimens of cancer-containing thyroid).

\section{Results}

Cancer and not cancer (i.e., normal tissue) regions of interest (ROIs) were delineated by a pathologist in specimens of thyroid glands and lymph nodes derived from 11 patients with papillary thyroid cancer (samples p1 through p11). Spectra generated by imaging of tryptic peptides were exported from both types of cancer ROIs - primary tumors in the thyroid gland and their metastases in lymph nodes, and both types of not cancer ROIs - normal thyroid glands and normal lymph nodes. The participation of spectra from each ROI in global figures was as follows: tumors-24.6\%, metastases- $4.2 \%$, normal thyroid $-45.1 \%$, and lymph nodes-26.1\% (yet variation was observed between individual samples).

In the first step, spectra from cancer ROIs (both types of cancer ROI from all specimens together) were clustered using unsupervised deglomerative image segmentation. The first step of segmentation revealed two clusters presented in Fig. 1a (clusters marked in green and red, respectively). The contribution of each cluster in either primary tumor or metastasis ROI from each patient was not random: the overrepresentation of cluster 1 (green) in primary tumor ROI and the overrepresentation of cluster 2 (red) in metastasis ROI was generally observed. Substantial contribution of cluster 2 in tumor ROI (approx. 70\%) was visible only for samples p2 and p7, while the substantial contribution of cluster 1 in metastasis ROI (approx. 50\%) was visible only for sample 11 (Fig. 1b). The contribution of each cluster in both ROIs was also calculated for all patients' samples together. The majority of spectra in primary tumor ROI belonged to cluster $1(67 \%)$ while the majority of spectra in metastasis ROI belonged to cluster 2 (89\%) (Fig. 1c). Hence, one could conclude the different molecular composition of cancer ROIs between the primary tumor and its metastasis. Moreover, the results of image segmentation suggested inter-patient similarity of a specific type of cancer ROIs.

In the next step, similarities between spectra derived from different types of ROIs were addressed more specifically. The
Fig. 1 Unsupervised segmentation of tissue regions corresponding to cancer. a The first level of segmentation of cancer ROIs detected in the thyroid glands and lymph nodes of all eleven patients (p1-p11); two resulting clusters are marked on microscopic images in green and red. $\mathbf{b}$ Contribution of both clusters detected in panel $\mathrm{A}$ in both types of cancer ROIs ( $\mathrm{T}-$ primary tumor, $\mathrm{M}$-metastasis) in tissue specimens of individual patients. c Participation of image clusters (1 and 2) in cancer ROIs ( $\mathrm{T}$ and $\mathrm{M}$ ) in all combined tissue specimens

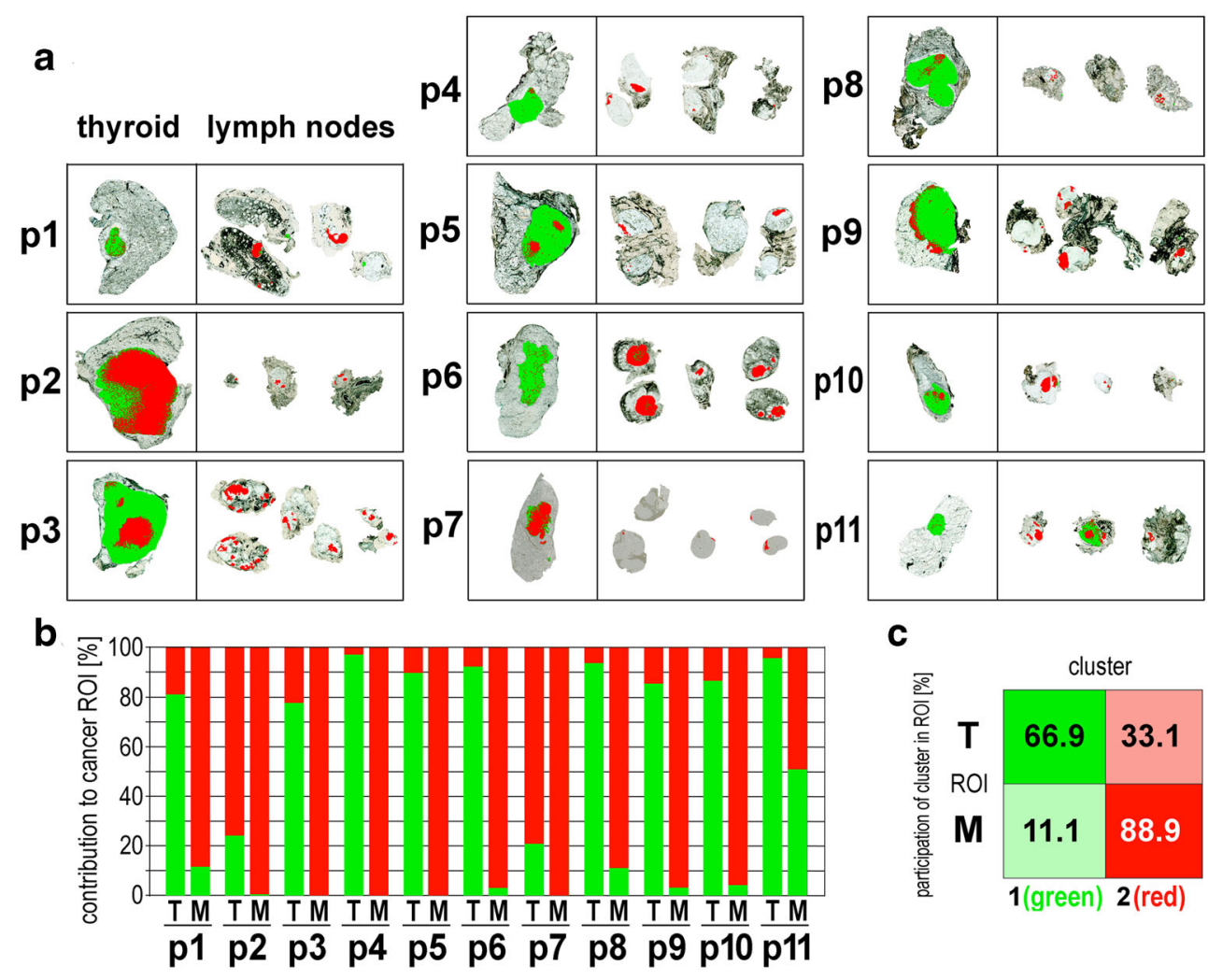


similarity index between pairwise compared spectra from four types of ROIs was estimated based on all registered molecular components (i.e., tryptic peptides); the frequencies of pairs with assumed similarity index and the resulting cumulative distribution functions are depicted in Fig. 2. To assess intratissue inter-patient heterogeneity, similarities between spectra from the same type of ROI were compared between different samples (patients). We found that the inter-patient heterogeneity was the highest for normal (not cancer) thyroid tissue and the lowest for normal lymph nodes, and that similarity between lymph node metastases of different patients was higher than the similarity between primary tumors of different patients (Fig. 2a). To assess inter-tissue intra-patient heterogeneity, similarities between spectra from different ROIs were compared within the same patient. We found that intra-patient heterogeneity was the highest between normal thyroid and normal lymph nodes while it was the lowest between normal lymph nodes and cancer metastases in lymph nodes. Moreover, the similarity between thyroid tumors and normal thyroids was higher than the similarity between thyroid tumors and their lymph node metastases (Fig. 2b). By direct comparison of similarities within and between both types of cancer ROIs, we stated that intra-tumor heterogeneity between thyroid tumors and their lymph node metastases in the same patient was higher than inter-cancer heterogeneity of thyroid tumors from different patients and cancer metastases
Fig. 2 Similarity index between different tissue regions. a Similarity between the same type of ROI assessed among different patients (inter-patient). b Similarity between different types of ROI assessed within the same patient (intra-patient). c Comparison of intra-patient similarity between two types of cancer ROIs (tumor and metastasis) and inter-patient similarity within the same type of cancer ROI (tumor or metastasis). The similarity index is presented as a relative number of events (left diagrams) and as a cumulative distribution function (right diagrams)
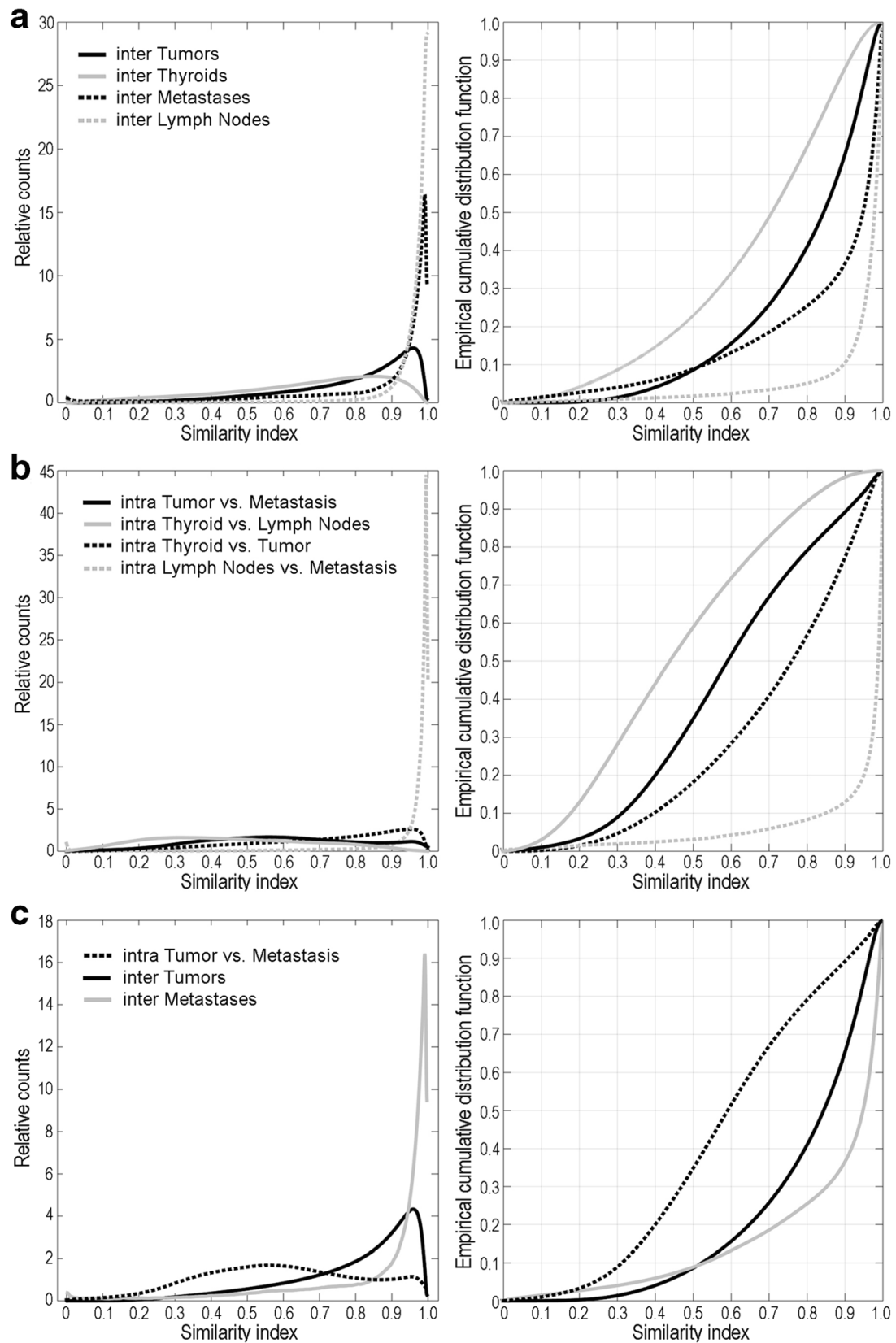
from different patients (the latter one was the most homogenous) (Fig. 2c). Hence, this observation was coherent with the results of the unsupervised segmentation of cancer ROIs presented above.

Finally, we searched for molecular components with abundances markedly different between different types of ROIs (all spectra from a given ROI were combined for analysis). Considering the structure of data and large disparity of the number of spectra across different ROIs, the strength of differences was estimated by the effect size factor, which is independent of the number of compared samples/spectra. Cohen's $d$ (absolute) values above $0.5,0.8$, and 1.2 corresponded to medium, large, and very large effects, respectively [30]. The number of components that discriminated different ROIs with different effect sizes is illustrated in Fig. 3 (see details in Supplementary Table S1). There were 96 components (ca. $4 \%$ of all registered components) whose abundances markedly differentiated normal thyroid gland and normal lymph nodes (including 15 components with a very large effect). However, relatively few components showed significantly different abundances between cancer regions and adjacent normal tissues. There were 29 components with markedly different abundances between thyroid tumor and normal thyroid (Table 1) and 17 components with markedly different abundances between metastases and normal lymph nodes (Table 2). On the other hand, a larger number of components showed markedly different abundances between thyroid tumors and their lymph node metastases. There were 36 components with markedly different abundances between thyroid tumors and their lymph node metastases. Importantly, most of them (32 components) similarly discriminated normal thyroid and lymph nodes (Table 3).

The hypothetical identity of MSI components could be established by attributing masses ( $\mathrm{m} / \mathrm{z}$ values) of imaged molecular components (i.e., tryptic peptides) to measured masses of peptides identified by the LC-MS/MS in lysates from the same type of tissue specimens. Here, hypothetical identity could be attributed to the majority of molecular components detected by MSI, yet one should be aware that this type of annotation is not unique and more than one identified peptide could be matched to an MSI component due to the relatively low resolution of MALDI-ToF MSI (Supplementary Table S2). Nevertheless, proteins whose tryptic fragments were the most frequently attributed to discriminatory MSI components included species involved in the development, homeostasis, cytoskeleton organization, extracellular matrix organization, chromatin organization, and cell death. Biological processes associated with hypothetical proteins discriminating between thyroid tumor and normal thyroid included gland development (THYG, APT, CAN1, HNRPD), chromosome organization (ACINU, DHX9, H2B2E, HNRPD, SKP1), and extracellular matrix organization
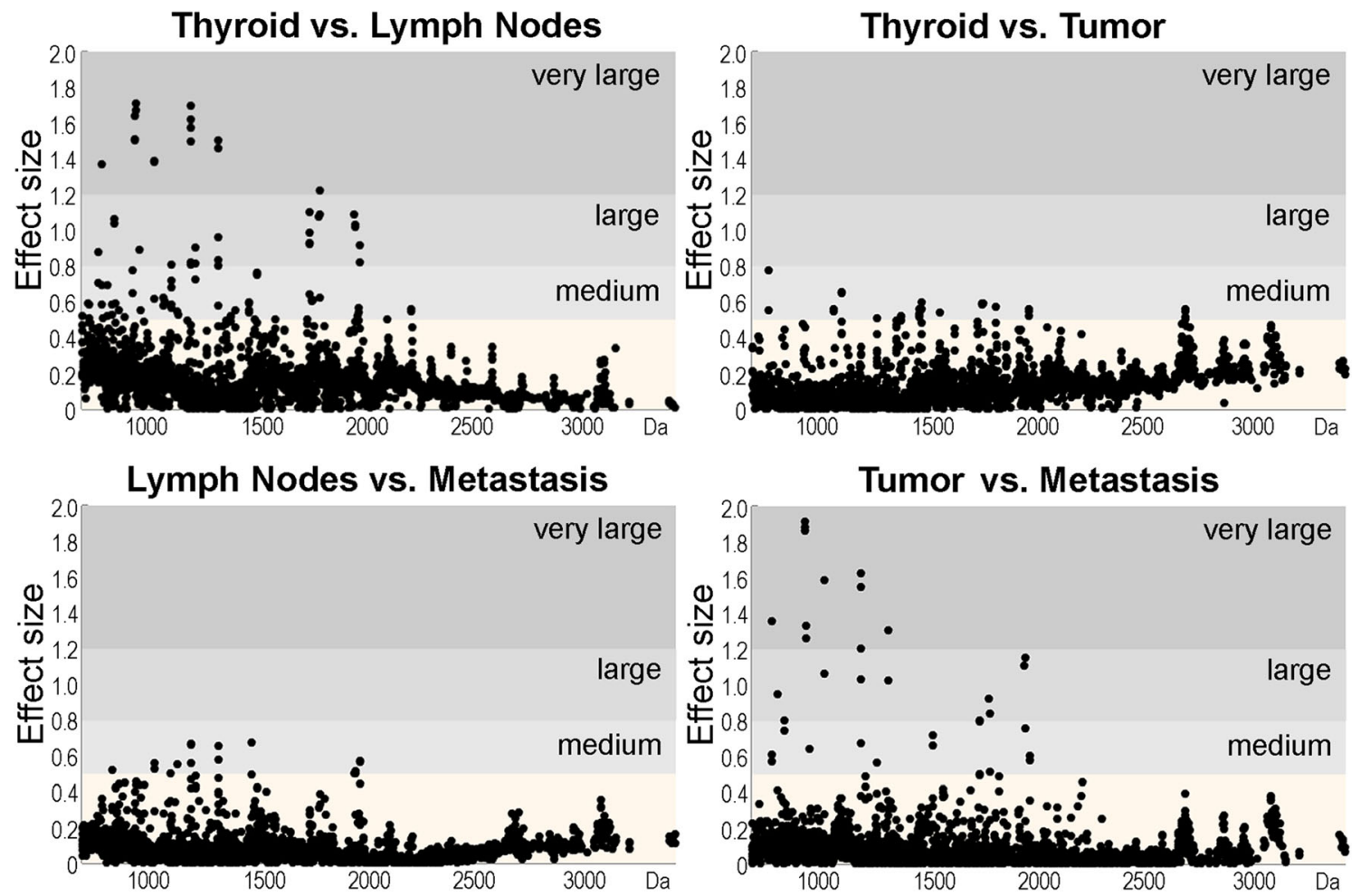

Fig. 3 Spectral components discriminating different types of ROIs. Significance of differences in the abundance of each component (represented by a dot) between ROIs was estimated as Cohen's $d$ effect

size (shown are absolute values); components are ordered according to their $\mathrm{m} / \mathrm{z}$ values 
Table 1 Components that discriminate between thyroid tumor and normal thyroid

\begin{tabular}{|c|c|c|c|}
\hline \multirow[t]{2}{*}{ Component $(\mathrm{m} / \mathrm{z})$} & \multicolumn{2}{|c|}{ Significance of differences (Cohen's effect size) } & \multirow[t]{2}{*}{ Hypothetical identity } \\
\hline & Tumor vs. thyroid & Tumor vs. metastasis & \\
\hline 775.49 & 0.775 & -0.103 & n.d. \\
\hline 776.50 & 0.554 & -0.121 & n.d. \\
\hline 1075.62 & 0.548 & 0.052 & CLUS, THYG, ANXA4 \\
\hline 1076.62 & 0.557 & -0.112 & GBRL3, LEG1, VIME \\
\hline 1111.54 & 0.656 & -0.033 & n.d. \\
\hline 1111.57 & 0.649 & 0.164 & n.d. \\
\hline 1274.72 & 0.508 & 0.565 & HBB, ARMX3, ACINU \\
\hline 1384.72 & 0.512 & 0.071 & THYG, NONO \\
\hline 1384.85 & 0.521 & 0.117 & \\
\hline 1465.68 & 0.537 & 0.104 & APT \\
\hline 1466.20 & 0.561 & -0.226 & SKP1, APT, TPIS1 \\
\hline 1466.68 & 0.522 & -0.103 & \\
\hline 1467.37 & 0.525 & -0.271 & CO6A3, ALBU \\
\hline 1467.68 & 0.514 & -0.207 & \\
\hline 1478.75 & -0.595 & -0.213 & TRFE, HBB, ODO2 \\
\hline 1479.75 & -0.559 & -0.316 & n.d. \\
\hline 1561.77 & -0.541 & -0.111 & ACTN4, OAT \\
\hline 1757.95 & 0.588 & -0.024 & THYG, CO6A1, EMIL1 \\
\hline 1758.91 & 0.586 & -0.028 & ACTN4, HNRPR \\
\hline 1758.95 & 0.590 & -0.031 & \\
\hline 1759.94 & 0.589 & -0.012 & $\mathrm{H} 2 \mathrm{~B} 2 \mathrm{E}$ \\
\hline 1818.86 & -0.574 & -0.283 & n.d. \\
\hline 1971.07 & 0.559 & 0.003 & DHX9, THYG \\
\hline 1972.05 & 0.548 & -0.035 & HNRPD \\
\hline 1973.05 & 0.523 & -0.056 & $\mathrm{SC} 22 \mathrm{~B}$ \\
\hline 2689.29 & -0.549 & -0.393 & SYSC, CAN1 \\
\hline 2690.31 & $-\mathbf{0 . 5 5 7}$ & -0.272 & \\
\hline 2692.37 & -0.513 & -0.273 & n.d. \\
\hline 2693.36 & -0.503 & -0.299 & n.d. \\
\hline
\end{tabular}

A positive value of the effect size indicates the factor's downregulation in the first ROI while its negative value indicates the factor's upregulation of the firsts ROI (significant differences are in bold, lack of significant differences in italics); listed are up to three proteins whose fragments showed the maximum concordance with $\mathrm{m} / \mathrm{z}$ values of imaged components (n.d. not determined) (collagens, CAN1, LEG1, HNRPR) (Table 1). Processes associated with hypothetical proteins discriminating between cancer metastasis and normal lymph nodes included actin cytoskeleton organization, hemostasis (ACTG, CO1A1, FIBB, $\mathrm{H} 33$, RAC2), and regulation of immune-related functions (ACTG, ACTR, CO1A1, FIBB, HCLS1, H4, K2C1, RAC2); importantly, an increased level of thyroglobulin (THYG) was characteristic for cancer metastasis when compared to normal lymph nodes (Table 2). Furthermore, processes associated with hypothetical proteins discriminating between thyroid tumor and their metastases included cytoskeleton organization (actins, keratins, CEP250, RAC2), chromosome organization (core histones), and blood-related functions/components (hemoglobin, fibrinogen, transferrin, CO4B) upregulated in lymph node metastases; importantly, the level of thyroglobulin did not discriminate between primary tumor and metastases (Table 3 ). On the other hand, thyroglobulin appeared the major protein discriminating normal thyroid from normal lymph nodes (11 out of 40 MSIdetected components upregulated in thyroid could be attributed to tryptic fragments of this thyroid-specific protein), while proteins involved in blood- and immune-related processes were overrepresented in normal thyroid (Supplementary Tables S1 and S2).

\section{Discussion}

In general, intra-tumor heterogeneity results from genetic heterogeneity of cancer (sub)clones and phenotypic plasticity induced among others by interactions between cancer cells and local microenvironment. Both components apparently affect the divergence between a primary tumor and metastatic outgrowths in lymph nodes, which is an important yet underresearched aspect of intra-tumor heterogeneity [1-3]. There are a few reports related to papillary thyroid cancer that addressed the divergence between a primary tumor and lymph node metastases, most of them concerning the status of BRAF V600E mutations. These works showed the concordance of a mutation status between the tumor and lymph node metastases in the majority of patients, yet cases with mutation-positive tumor and mutation-negative metastases or mutation-negative 
Table 2 Components that discriminate between cancer metastasis in lymph nodes and normal lymph nodes

\begin{tabular}{|c|c|c|c|}
\hline \multirow[t]{2}{*}{ Component $(\mathrm{m} / \mathrm{z})$} & \multicolumn{2}{|c|}{ Significance of differences (Cohen's effect size) } & \multirow[t]{2}{*}{ Hypothetical identity } \\
\hline & Metastasis vs. LN & Tumor vs. thyroid & \\
\hline 836.43 & -0.523 & 0.018 & CO1A1, RS10, SYNC1 \\
\hline 1032.57 & 0.524 & 0.143 & FIBB, RAC2, H33 \\
\hline 1033.58 & 0.560 & -0.027 & $\mathrm{AOC} 3, \mathrm{~K} 2 \mathrm{C} 1$ \\
\hline 1105.56 & -0.501 & -0.034 & DAAM2, THYG, PUF60 \\
\hline 1138.55 & -0.551 & 0.340 & SRSF9, EF2, SDHB \\
\hline 1199.71 & 0.556 & -0.306 & HSP7C \\
\hline 1200.70 & 0.667 & -0.307 & CPSF1 \\
\hline 1200.71 & 0.660 & -0.349 & \\
\hline 1325.73 & 0.578 & 0.115 & H4, HCLS1 \\
\hline 1326.73 & 0.652 & 0.001 & TKT \\
\hline 1477.74 & -0.673 & -0.482 & RINI, SDHF2, PEA15 \\
\hline 1953.13 & 0.505 & 0.098 & TRFE \\
\hline 1954.11 & 0.501 & 0.373 & ACTG, ACTBL, SEPT7 \\
\hline 1954.12 & 0.502 & 0.373 & \\
\hline 1955.10 & 0.514 & 0.152 & $\mathrm{KCD} 12, \mathrm{ACTC}$ \\
\hline 1976.03 & 0.568 & 0.121 & ACTA \\
\hline 1977.04 & 0.575 & 0.034 & ARP2, ACTA \\
\hline
\end{tabular}

A positive value of the effect size indicates the factor's downregulation in the first ROI while its negative value indicates the factor's upregulation of the firsts ROI (significant differences are in bold, lack of significant differences in italics); listed are up to three proteins whose fragments showed the maximum concordance with $\mathrm{m} / \mathrm{z}$ values of imaged components (n.d. not determined) tumor and mutation-positive metastases were also frequently reported $[13,14,34,35]$. An interesting genomics study was reported by Le Pennec et al. [15] who performed a systemic cDNA sequencing screening of a primary tumor (different subregions), lymph node metastases and distant (pleural) metastasis in a patient with an aggressive PTC. The study revealed the existence of several cancer subclones and showed a greater genetic divergence between a primary tumor and lymph node metastases than between a primary tumor and distant metastasis. Moreover, differences between metastases in two different lymph nodes were detected (though it should be noted that the material from metastases was collected after radioiodine treatment of the patient) [15]. These genetic data collectively confirmed the subclonal appearance of mutational events during PTC tumorigenesis. However, a phenotypic molecular divergence between a primary tumor and its metastases was not addressed systematically in PTC samples yet.

Mass spectrometry imaging, which is a perfect tool to analyze molecular tissue heterogeneity, was used in several studies focused on the classification of different types of thyroid tumors, e.g., [36-38]. Here, we used this approach to compare for the first time the molecular profile of PTC in a primary tumor site (i.e., thyroid gland), and its lymph node metastases aimed to estimate inter-patient/tumor and intra-patient/tumor heterogeneity of these two cancer regions. We found a higher level of molecular similarity amongst thyroid tumors than amongst "normal" thyroid tissue from different patients. A similar observation was reported previously when lipid component was imaged by MALDI-MSI in a tumor and adjacent not cancerous thyroid tissue of PTC patients [39]. These interesting observations could be related to the fact that different pathological conditions (e.g., inflammation-related) frequently exist in not cancerous tissue adjacent to a tumor that was considered here as a "normal thyroid." Moreover, we observed several differences between primary tumors and their lymph node metastases. Interestingly, the intra-tumor heterogeneity between primary tumors and metastases from the same patient was higher than the inter-tumor heterogeneity between primary tumors from different patients. This intriguing phenomenon apparently mirrored changes in a phenotype of cancer cells induced by a specific lymph node microenvironment and/or specific phenotypic features of invasive cancer cells. Lymphocytes and other blood cells are the major components of lymph nodes; hence, the cross-talk between immune cells and cancer cells should be the critical factor affecting the phenotype of cancer cells located in this organ. Classical hallmarks of cancer include immune-related mechanisms; evading immune destruction and tumor-promoting inflammation [4]. It is well known that, in addition to the suppression of anti-tumor functions of the immune system by progressing cancer, immune cells promote cancer progression in many ways. These not only include remodeling of tumor 
Table 3 Components that discriminate between thyroid tumor and its metastasis in lymph nodes

\begin{tabular}{|c|c|c|c|}
\hline \multirow[t]{2}{*}{ Component $(\mathrm{m} / \mathrm{z})$} & \multicolumn{2}{|c|}{ Significance of differences (Cohen's effect size) } & \multirow[t]{2}{*}{ Hypothetical identity } \\
\hline & Tumor vs. metastasis & Thyroid vs. LN & \\
\hline 788.47 & 1.358 & 1.371 & n.d. \\
\hline 788.99 & 0.611 & 0.582 & n.d. \\
\hline 789.47 & 0.573 & 0.694 & n.d. \\
\hline 816.44 & 0.952 & 0.693 & H2B1B, CP250, FBN1 \\
\hline 850.49 & 0.743 & 1.039 & GRD2I, K1C19, H2AX \\
\hline 850.51 & 0.803 & 1.066 & \\
\hline 944.52 & 1.879 & 1.503 & $\mathrm{H} 2 \mathrm{AX}$ \\
\hline 944.74 & 1.863 & 1.509 & \\
\hline 945.52 & 1.913 & 1.641 & TIM10, PGBM, MATR3 \\
\hline 946.51 & 1.259 & 1.673 & n.d. \\
\hline 946.52 & 1.330 & 1.709 & n.d. \\
\hline 966.50 & 0.644 & 0.889 & RP10A \\
\hline 1032.57 & 1.590 & 1.387 & FIBB, RAC2, H33 \\
\hline 1033.58 & 1.065 & 1.385 & $\mathrm{AOC} 3, \mathrm{~K} 2 \mathrm{C} 1$ \\
\hline 1198.71 & 1.624 & 1.498 & ACTC, POTEE, \\
\hline 1198.91 & 0.675 & 0.819 & \\
\hline 1199.71 & 1.547 & 1.576 & HSP7C, ACTN \\
\hline 1200.70 & 1.029 & 1.622 & CPSF1 \\
\hline 1200.71 & 1.202 & 1.697 & \\
\hline 1274.72 & 0.565 & -0.071 & H2AX, HBB, TCPE \\
\hline 1325.73 & 1.304 & 1.504 & H4, HCLS1 \\
\hline 1326.73 & 1.028 & 1.462 & TKT \\
\hline 1529.70 & 0.659 & 0.399 & HBA, TYPH \\
\hline 1530.70 & 0.718 & 0.364 & G3P \\
\hline 1743.77 & 0.802 & 0.924 & $\mathrm{H} 2 \mathrm{~B} 1 \mathrm{~B}, \mathrm{CO} 6 \mathrm{~A} 3, \mathrm{CO} 4 \mathrm{~B}$ \\
\hline 1743.80 & 0.802 & 0.928 & \\
\hline 1744.79 & 0.795 & 1.100 & ACTG \\
\hline 1745.78 & 0.503 & 0.988 & HSP7C \\
\hline 1790.88 & 0.925 & 1.077 & ACTG, PTPRC \\
\hline 1791.88 & 0.843 & 1.224 & $\mathrm{~K} 1 \mathrm{C} 9$ \\
\hline 1792.88 & 0.515 & 1.091 & MATR3 \\
\hline 1953.13 & 1.110 & 1.092 & TRFE \\
\hline 1954.11 & 1.155 & 1.030 & SEPT7, ACTG, KCD12 \\
\hline 1954.12 & 1.154 & 1.031 & \\
\hline 1955.10 & 0.756 & 1.016 & GPX3 \\
\hline 1976.03 & 0.575 & 0.328 & ACTA \\
\hline 1977.04 & 0.602 & 0.916 & ACTR \\
\hline
\end{tabular}

A positive value of the effect size indicates the factor's downregulation in the first ROI while its negative value indicates the factor's upregulation of the firsts ROI (significant differences are in bold, lack of significant differences in italics); listed are up to three proteins whose fragments showed the maximum concordance with $\mathrm{m} / \mathrm{z}$ values of imaged components (n.d. not determined) niche (e.g., reorganization of extracellular matrix and formation of blood vessels) but also production of cytokines and growth factors that increase motility and invasiveness of cancer cells as well as decrease their sensitivity to pro-death factors $[40,41]$. Immune cells have an obvious role also in the development and progression of thyroid cancers [42, 43]. On the other hand, phenotypic differences between a primary tumor and its lymph node metastases could also reflect invasionrelated features of metastatic cells. Increased motility and invasiveness of cancer cells involves remodeling of their skeleton and extracellular matrix; hence, factors regulating these cellular components are frequently associated with the 
aggressiveness of thyroid cancer [44, 45]. Moreover, mechanisms related to epithelial to mesenchymal transition, possibly involving cancer stem-like cells, are well documented in the progression of thyroid cancers $[46,47]$. The strong influence of the tumor niche in lymph nodes and specific features associated with invasiveness could explain the relative "equalization" of a phenotype of metastatic cancer cells since interpatient heterogeneity of metastases was markedly lower than inter-patient heterogeneity of thyroid tumors. Finally, hypothetical coexistence of cancer cells and immune cells in tissue regions delineated as metastasis ROI could also contribute to differences observed between a primary tumor and metastasis ROIs as well as to similarities observed between metastasis and lymph node ROIs.

Overall similarities between tissue regions were obviously associated with the number of components that showed significantly different abundances between these regions. The highest number of discriminatory components was observed between the most dissimilar tissues - normal thyroid and normal lymph nodes. As one could expect, components more abundant in lymph nodes were hypothetically attributed to proteins involved in immune-related functions and blood components. On the other hand, the major factor specific for thyroid (as well as for both types of cancer regions) was thyroglobulin, the precursor of thyroid hormones which is the major protein synthesized in this gland. Components that differentiated normal and cancerous thyroid included those attributed to proteins involved in gland development and functions (e.g., thyroglobulin) as well as in chromosome organization and extracellular matrix organization, factors apparently associated with the etiology of thyroid cancer. In fact, changes in the chromosome structure are frequently observed in thyroid cancers and the abnormal nuclear morphology is an important diagnostic factor in PTC [48]. Remodeling of the extracellular matrix is associated with the development and progression of different malignancies [49] and differential expression of its components (e.g., galectins and collagens) was reported also in PTC $[50,51]$. It is noteworthy that the same components that discriminated normal and cancerous thyroid glands did not discriminate the thyroid tumor and its metastases in lymph nodes, which suggested their general cancerspecific patterns. On the other hand, there were two types of features differentiating tumor and its metastases. One of them included proteins involved in immune-related functions and other blood components, which most probably reflected the infiltration of immune cells in metastasis ROIs. Another group of differentiating features included chromatin proteins (e.g., core histones) and proteins involved in the cytoskeleton organization (e.g., actins and keratins). Noteworthy, demethylation and other epigenetic modifications of core histones were reported as an important factor in early lymphatic metastasis of PTC that resulted in modulation of migration and invasiveness of cancer cells [52]. Moreover, remodeling of the cytoskeleton was generally involved in the epithelial-mesenchymal transition and metastatic potential of cancer cells [53]. Interestingly, different properties of actin cytoskeleton were reported for colorectal cancer cells derived from the primary tumor and its lymph node metastasis from the same patient [54], yet similar data is not available for thyroid cancer. It is also noteworthy that cytokeratin 19 (K1C19; upregulated here in metastasis ROI), an established marker of different malignancies, was highly expressed in lymph node metastases of PTC [55] and was associated with extensive vascular invasion of follicular thyroid cancer [56]. Even though a relative overall similarity between lymph nodes and cancer metastases in lymph nodes was noted, several components discriminating these ROIs were detected. These included thyroglobulin detected in cancer metastasis as well as proteins involved in functions of blood and immune cells detected in normal lymph nodes (not surprisingly, similar components discriminated between normal thyroid and normal lymph nodes). In general, proteins hypothetically ascribed to components that discriminated different ROIs reflected molecular features and functions that could be attributed to the imaged types of tissue. However, this type of analysis was not specific enough to identify features of cancer proteome associated with invasive potential, hence preferably observed in metastatic cells, or their changes related to the influence of lymph node microenvironment (mainly interactions between cancer cells and immune cells).

\section{Conclusions}

A marked molecular difference between the primary thyroid cancer and its lymph node metastases was observed using mass spectrometry imaging. Importantly, we concluded that phenotypical inter-tumor heterogeneity between primary tumors from different patients was lower than intra-tumor heterogeneity between primary tumor and lymph node metastases from the same patient.

Authors' Contributions PW contributed to the conceptualization. PW and JP helped obtain the funding. MG and MP helped in the development of experimental methodology. JP contributed to the development and implementation of algorithms. MG, AK, ES, and MP contributed to the investigation. $\mathrm{KF}$ contributed to the spectra preprocessing and signal modeling. AK contributed to the formal analysis. PW wrote the manuscript. All authors read and approved the final manuscript.

Funding Information This work was financially funded by the National Science Centre, Poland, grant 2016/23/B/NZ4/03901 (to P.W.) and grant 2015/19/B/ST6/01736 (to J.P.), and the National Centre for Research and Development, Poland, grant DZP/STRATEGMED2/2554/2014 (to P.W.). K.F. was funded by BKM grant (Silesian University of Technology) no. 02/010/BKM18/0136 and by the European Union through the European Social Fund grant POWR.03.02.00-00-I02. 


\section{Compliance with Ethical Standards}

Conflict of Interest Statement The authors declare no conflict of interest.

Open Access This article is distributed under the terms of the Creative Commons Attribution 4.0 International License (http:// creativecommons.org/licenses/by/4.0/), which permits unrestricted use, distribution, and reproduction in any medium, provided you give appropriate credit to the original author(s) and the source, provide a link to the Creative Commons license, and indicate if changes were made.

\section{References}

1. Nowell PC (1976) The clonal evolution of tumor cell populations. Science. 194:23-28. https://doi.org/10.1126/science.959840

2. Greaves M, Maley CC (2012) Clonal evolution in cancer. Nature. 481:306-313. https://doi.org/10.1038/nature10762

3. Marusyk A, Almendro V, Polyak K (2012) Intra-tumour heterogeneity: a looking glass for cancer? Nat Rev Cancer. 12:323-334. https://doi.org/10.1038/nrc3261

4. Hanahan D, Weinberg RA (2011) Hallmarks of cancer: the next generation. Cell. 144:646-674. https://doi.org/10.1016/j.cell.2011. 02.013

5. Ramaswamy S, Ross KN, Lander ES, Golub TR (2003) A molecular signature of metastasis in primary solid tumors. Nat Genet. 33: 49-54. https://doi.org/10.1038/ng1060

6. Stoecklein NH, Klein CA (2010) Genetic disparity between primary tumours, disseminated tumour cells, and manifest metastasis. Int J Cancer. 126:589-598. https://doi.org/10.1002/ijc.24916

7. Ding L, Ellis MJ, Li S, Larson DE, Chen K, Wallis JW, Harris CC, McLellan M, Fulton RS, Fulton LL, Abbott RM, Hoog J, Dooling DJ, Koboldt DC, Schmidt H, Kalicki J, Zhang Q, Chen L, Lin L, Wendl MC, McMichael J, Magrini VJ, Cook L, McGrath S, Vickery TL, Appelbaum E, Deschryver K, Davies S, Guintoli T, Lin L, Crowder R, Tao Y, Snider JE, Smith SM, Dukes AF, Sanderson GE, Pohl CS, Delehaunty KD, Fronick CC, Pape KA, Reed JS, Robinson JS, Hodges JS, Schierding W, Dees ND, Shen D, Locke DP, Wiechert ME, Eldred JM, Peck JB, Oberkfell BJ, Lolofie JT, du F, Hawkins AE, O'Laughlin MD, Bernard KE, Cunningham M, Elliott G, Mason MD, Thompson DM Jr, Ivanovich JL, Goodfellow PJ, Perou CM, Weinstock GM, Aft R, Watson M, Ley TJ, Wilson RK, Mardis ER (2010) Genome remodelling in a basal-like breast cancer metastasis and xenograft. Nature. 464:999-1005. https://doi.org/10.1038/nature08989

8. Brannon AR, Vakiani E, Sylvester BE, et al. (2014) Comparative sequencing analysis reveals high genomic concordance between matched primary and metastatic colorectal cancer lesions. Genome Biol. 15:454. https://doi.org/10.1186/s13059-014-0454-7

9. Sleeman JP, Cady B, Pantel K (2012) The connectivity of lymphogenous and hematogenous tumor cell dissemination:biological insights and clinical implications. Clin Exp Metastasis. 29:737746. https://doi.org/10.1007/s10585-012-9489-x

10. Knösel T, Schlüns K, Stein U, et al. (2004) Chromosomal alterations during lymphatic and liver metastasis formation of colorectal cancer. Neoplasia. 6:23-28. https://doi.org/10.1016/s14765586(04)80050-2

11. Sylvester BE, Vakiani E (2015) Tumor evolution and intratumor heterogeneity in colorectal carcinoma: insights from comparative genomic profiling of primary tumors and matched metastases. J Gastrointest Oncol. 6:668-675. https://doi.org/10.3978/j.issn.20786891.2015 .083
12. Anaka M, Hudson C, Lo PH, Do H, Caballero OL, Davis ID, Dobrovic A, Cebon J, Behren A (2013) Intratumoral genetic heterogeneity in metastatic melanoma is accompanied by variation in malignant behaviors. BMC Med Genomics. 6:40. https://doi.org/ 10.1186/1755-8794-6-40, 13

13. Giannini R, Ugolini C, Lupi C, Proietti A, Elisei R, Salvatore G, Berti P, Materazzi G, Miccoli P, Santoro M, Basolo F (2007) The heterogeneous distribution of BRAF mutation supports the independent clonal origin of distinct tumor foci in multifocal papillary thyroid carcinoma. J Clin Endocrinol Metab. 92:3511-3516. https:// doi.org/10.1210/jc.2007-0594

14. Walts AE, Pao A, Sacks W, Bose S (2014) BRAF genetic heterogeneity in papillary thyroid carcinoma and its metastasis. Hum Pathol. 45:935-941. https://doi.org/10.1016/j.humpath.2013.12. 005

15. Le Pennec S, Konopka T, Gacquer D et al. (2015) Intratumor heterogeneity and clonal evolution in an aggressive papillary thyroid cancer and matched metastases. Endocr Relat Cancer. 22:205-216. https://doi.org/10.1530/ERC-14-0351

16. Santinelli A, Pisa E, Stramazzotti D, Fabris G (2008) HER-2 status discrepancy between primary breast cancer and metastatic sites. Impact on target therapy. Int J Cancer. 122:999-1004. https://doi. org/10.1002/ijc. 23051

17. Gerlinger M, Rowan AJ, Horswell S, Math M, Larkin J, Endesfelder D, Gronroos E, Martinez P, Matthews N, Stewart A, Tarpey P, Varela I, Phillimore B, Begum S, McDonald N, Butler A, Jones D, Raine K, Latimer C, Santos CR, Nohadani M, Eklund AC, Spencer-Dene B, Clark G, Pickering L, Stamp G, Gore M, Szallasi Z, Downward J, Futreal PA, Swanton C (2012) Intratumor heterogeneity and branched evolution revealed by multiregion sequencing. N Engl J Med. 366:883-892. https://doi.org/10.1056/ NEJMoa1113205

18. Navin N, Kendall J, Troge J, Andrews P, Rodgers L, McIndoo J, Cook K, Stepansky A, Levy D, Esposito D, Muthuswamy L, Krasnitz A, McCombie W, Hicks J, Wigler M (2011) Tumour evolution inferred by single-cell sequencing. Nature. 472:90-94. https://doi.org/10.1038/nature09807

19. Cornett DS, Reyzer ML, Chaurand P, Caprioli RM (2007) MALDI imaging mass spectrometry: molecular snapshots of biochemical systems. Nat Methods. 4:828-833. https://doi.org/10.1038/ nmeth1094

20. McDonnell LA, Heeren RM (2007) Imaging mass spectrometry. Mass Spectrom Rev. 26:606-643. https://doi.org/10.1002/mas. 20124

21. Schwamborn K, Caprioli RM (2010) Molecular imaging by mass spectrometry-looking beyond classical histology. Nat Rev Cancer. 10:639-646. https://doi.org/10.1038/nrc2917

22. Aichler M, Walch A (2015) MALDI Imaging mass spectrometry: current frontiers and perspectives in pathology research and practice. Lab Invest. 95:422-431. https://doi.org/10.1038/labinvest. 2014.156

23. Heijs B, Carreira RJ, Tolner EA, et al. (2015) Comprehensive analysis of the mouse brain proteome sampled in mass spectrometry imaging. Anal. Chem. 87:1867-1875. https://doi.org/10.1021/ ac503952q

24. Bednarczyk K, Gawin M, Chekan M, Kurczyk A, Mrukwa G, Pietrowska M, Polanska J, Widlak P (2019) Discrimination of normal oral mucosa from oral cancer by mass spectrometry imaging of proteins and lipids. J Mol Histol. 50:1-10. https://doi.org/10.1007/ s10735-018-9802-3

25. Bruffaerts C, Verardi V, Vermandele C (2014) A generalized boxplot for skewed and heavy-tailed distributions. Stat Probab Lett. 95: 110-117. https://doi.org/10.1016/j.spl.2014.08.016

26. Wong JWH, Durante C, Cartwright HM (2005) Application of fast Fourier transform cross-correlation for the alignment of large 
chromatographic and spectral datasets. Anal Chem. 77:5655-5661. https://doi.org/10.1021/ac050619p

27. Polanski A, Marczyk M, Pietrowska M, Widlak P, Polanska J (2015) Signal partitioning algorithm for highly efficient gaussian mixture modeling in mass spectrometry. PLoS ONE. 10: e0134256. https://doi.org/10.1371/journal.pone.013425

28. Polanski A, Marczyk M, Pietrowska M, Widlak P, Polanska J (2018) Initializing EM algorithm for univariate Gaussian, multicomponent, heteroscedastic mixture models by dynamic programming partitions, Int J Comput Methods. 15:e1850012. https://doi. org/10.1142/S0219876218500123

29. Frank AM, Bandeira N, Shen Z (2008) Clustering millions of tandem mass spectra research articles. J Proteome Res. 7:113-122. https://doi.org/10.1021/pr070 361e

30. Cohen J (1988) Statistical Power Analysis for the Behavioral Sciences; 2nd ed.; New Jersey: Lawrence Erlbaum Associates

31. Widlak P, Mrukwa G, Kalinowska M, et al. (2016) Detection of molecular signatures of oral squamous cell carcinoma and normal epithelium - application of a novel methodology for unsupervised segmentation of imaging mass spectrometry data. Proteomics. 16: 1613-1621. https://doi.org/10.1002/pmic.201500458

32. Mrukwa G, Drazek G, Pietrowska M, Widlak P, Polanska J (2016) Novel divisive iK-means algorithm with region-driven feature selection as a tool for automated detection of tumour heterogeneity in MALDI IMS experiments. In: Bioinformatics and Biomedical Engineering. IWBBIO 2016. Lecture Notes in Computer Science. Ed. Ortuño F, Rojas I. Springer Cham, pp 113-124

33. Gawin M, Wojakowska A, Pietrowska M (2018) Proteome profiles of different types of thyroid cancers. Mol Cell Endocrinol. 474:6879. https://doi.org/10.1016/j.mce.2017.11.020

34. Kurtulmus N, Ertas B, Saglican Y, Kaya H, Ince U, Duren M (2016) BRAFV600E Mutation: Has It a Role in Cervical Lymph Node Metastasis of Papillary Thyroid Cancer? Eur Thyroid J. 5:195200. https://doi.org/10.1159/000448112

35. Shifrin AL, Fischer M, Paul T, et al. (2017) Mutational analysis of metastatic lymph nodes from papillary thyroid carcinoma in adult and pediatric patients. Surgery. 161:176-187. https://doi.org/10. 1016/j.surg.2016.10.002

36. Pagni F, De Sio G, Garancini M, et al. (2016) Proteomics in thyroid cytopathology: Relevance of MALDI-imaging in distinguishing malignant from benign lesions. Proteomics. 16:1775-1784. https:// doi.org/10.1002/pmic.201500448

37. Pietrowska M, Diehl HC, Mrukwa G, Kalinowska-Herok M, Gawin M, Chekan M, Elm J, Drazek G, Krawczyk A, Lange D, Meyer HE, Polanska J, Henkel C, Widlak P (2017) Molecular profiles of thyroid cancer subtypes: Classification based on features of tissue revealed by mass spectrometry imaging. Biochim Biophys Acta Proteins Proteom. 1865:837-845. https://doi.org/10.1016/j. bbapap.2016.10.006

38. Galli M, Pagni F, De Sio G, et al. (2017) Proteomic profiles of thyroid tumors by mass spectrometry-imaging on tissue microarrays. Biochim Biophys Acta Proteins Proteom. 1865:817-827. https://doi.org/10.1016/j.bbapap.2016.11.020

39. Wojakowska A, Cole LM, Chekan M, Bednarczyk K, Maksymiak M, Oczko-Wojciechowska M, Jarząb B, Clench MR, Polańska J, Pietrowska M, Widlak P (2018) Discrimination of papillary thyroid cancer from non-cancerous thyroid tissue based on lipid profiling by mass spectrometry imaging. Endokrynol Pol. 69:2-8. https://doi. org/10.5603/EP.a2018.0003

40. Kitamura T, Qian BZ, Pollard JW (2015) Immune cell promotion of metastasis. Nat Rev Immunol. 15:73-86. https://doi.org/10.1038/ nri3789

41. Janssen LME, Ramsay EE, Logsdon CD, Overwijk WW (2017) The immune system in cancer metastasis: friend or foe? $\mathrm{J}$ Immunother Cancer. 5:79. https://doi.org/10.1186/s40425-0170283-9
42. Galdiero MR, Varricchi G, Marone G (2016) The immune network in thyroid cancer. Oncoimmunology. 5:e1168556. https://doi.org/ 10.1080/2162402X.2016.1168556

43. Mould RC, van Vloten JP, AuYeung AWK, Karimi K, Bridle BW (2017) Immune responses in the thyroid cancer microenvironment: making immunotherapy a possible mission. Endocr Relat Cancer. 24:T311-T329. https://doi.org/10.1530/ERC-17-0316

44. Montero-Conde C, Martín-Campos JM, Lerma E, Gimenez G, Martínez-Guitarte JL, Combalía N, Montaner D, Matías-Guiu X, Dopazo J, de Leiva A, Robledo M, Mauricio D (2008) Molecular profiling related to poor prognosis in thyroid carcinoma. Combining gene expression data and biological information. Oncogene. 27:1554-1561. https://doi.org/10.1038/sj.onc.1210792

45. Ringel MD (2009) Molecular markers of aggressiveness of thyroid cancer. Curr Opin Endocrinol Diabetes Obes. 16:361-366. https:// doi.org/10.1097/MED.0b013e32832ff2cb

46. Hardin H, Zhang R, Helein H, Buehler D, Guo Z, Lloyd RV (2017) The evolving concept of cancer stem-like cells in thyroid cancer and other solid tumors. Lab Invest. 97:1142-1151. https://doi.org/10. 1038/labinvest.2017.41

47. Revilla G, Corcoy R, Moral A, Escolà-Gil JC, Mato E (2019) Cross-talk between Inflammatory Mediators and the epithelial mesenchymal transition process in the development of thyroid carcinoma. Int J Mol Sci. 20:e2466. https://doi.org/10.3390/ijms20102466

48. Caria P, Vanni R (2010) Cytogenetic and molecular events in adenoma and well-differentiated thyroid follicular-cell neoplasia. Cancer Genet Cytogenet. 203:21-29. https://doi.org/10.1016/j. cancergencyto.2010.08.025

49. Bonnans C, Chou J, Werb Z (2014) Remodelling the extracellular matrix in development and disease. Nat Rev Mol Cell Biol. 15:786801. https://doi.org/10.1038/nrm3904

50. Bartolazzi A, Sciacchitano S, D'Alessandria C (2018) Galectin-3: the impact on the clinical management of patients with thyroid nodules and future perspectives. Int J Mol Sci. 19:e445. https:// doi.org/10.3390/ijms19020445

51. Huang C, Yang X, Han L, Fan Z, Liu B, Zhang C, Lu T (2019) The prognostic potential of alpha-1 type I collagen expression in papillary thyroid cancer. Biochem Biophys Res Commun. 515:125-132. https://doi.org/10.1016/j.bbrc.2019.04.119

52. Zhang W, Sun W, Qin Y, et al. (2019) Knockdown of KDM1A suppresses tumour migration and invasion by epigenetically regulating the TIMP1/MMP9 pathway in papillary thyroid cancer. J Cell Mol Med. https://doi.org/10.1111/jcmm.14311

53. Yilmaz M, Christofori G (2009) EMT, the cytoskeleton, and cancer cell invasion. Cancer Metastasis Rev. 28:15-33. https://doi.org/10. 1007/s10555-008-9169-0

54. Palmieri V, Lucchetti D, Maiorana A, et al. (2015) Mechanical and structural comparison between primary tumor and lymph node metastasis cells in colorectal cancer. Soft Matter. 11:5719-5726. https://doi.org/10.1039/c5sm01089f

55. del Carmen S, Gatius S, Franch-Arcas G, Baena JA, Gonzalez O, Zafon C, Cuevas D, Valls J, Pérez A, Martinez M, Ros S, Macías CG, Iglesias C, Matías-Guiu X, de Álava E (2016) Concordance study between one-step nucleic acid amplification and morphologic techniques to detect lymph node metastasis in papillary carcinoma of the thyroid. Hum Pathol. 48:132-141. https://doi.org/10.1016/j. humpath.2015.09.020

56. Lee YM, Song DE, Kim TY, et al. (2016) Risk factors for distant metastasis in patients with minimally invasive follicular thyroid carcinoma. PLoS One. 11:e0155489. https://doi.org/10.1371/ journal.pone. 015548

Publisher's Note Springer Nature remains neutral with regard to jurisdictional claims in published maps and institutional affiliations. 